\title{
Azacitidine improves clinical outcomes in older patients with acute myeloid leukaemia with myelodysplasia-related changes compared with conventional care regimens
}

John F. Seymour ${ }^{1,2^{*}}$ (D), Hartmut Döhner ${ }^{3}$, Aleksandra Butrym ${ }^{4}$, Agnieszka Wierzbowska ${ }^{5}$, Dominik Selleslag ${ }^{6}$, Jun Ho Jang ${ }^{7}$, Rajat Kumar ${ }^{8}$, James Cavenagh ${ }^{9}$, Andre C. Schuh ${ }^{10}$, Anna Candoni ${ }^{11}$, Christian Récher ${ }^{12}$, Irwindeep Sandhu ${ }^{13}$, Teresa Bernal del Castillo ${ }^{14}$, Haifa Kathrin Al-Ali ${ }^{15}$, Jose Falantes ${ }^{16}$, Richard M. Stone ${ }^{17}$, Mark D. Minden ${ }^{10}$, Jerry Weaver ${ }^{18}$, Steve Songer ${ }^{18}$, C. L. Beach $^{18}$ and Hervé Dombret ${ }^{19}$

\footnotetext{
Abstract

Background: Compared with World Health Organization-defined acute myeloid leukaemia (AML) not otherwise specified, patients with AML with myelodysplasia-related changes (AML-MRC) are generally older and more likely to have poor-risk cytogenetics, leading to poor response and prognosis. More than one-half of all older ( $\geq 65$ years) patients in the phase 3 AZA-AML-001 trial had newly diagnosed AML-MRC.

Methods: We compared clinical outcomes for patients with AML-MRC treated with azacitidine or conventional care regimens (CCR; induction chemotherapy, low-dose cytarabine, or supportive care only) overall and within patient subgroups defined by cytogenetic risk (intermediate or poor) and age (65-74 years or $\geq 75$ years). The same analyses were used to compare azacitidine with low-dose cytarabine in patients who had been preselected to lowdose cytarabine before they were randomized to receive azacitidine or CCR (ie, low-dose cytarabine).

(Continued on next page)
}

\footnotetext{
* Correspondence: john.seymour@petermac.org

${ }^{1}$ Department of Haematology, Peter MacCallum Cancer Centre, Locked Bag

1, A'Beckett St, East Melbourne, VIC 8006, Australia

${ }^{2}$ University of Melbourne, Parkville, Australia

Full list of author information is available at the end of the article
} 
(Continued from previous page)

Results: Median overall survival was significantly prolonged with azacitidine $(n=129)$ versus CCR $(n=133)$ : 8.9 versus 4.9 months (hazard ratio $0.74,[95 \% \mathrm{Cl} 0.57,0.97]$ ). Among patients with intermediate-risk cytogenetics, median overall survival with azacitidine was 16.4 months, and with CCR was 8.9 months (hazard ratio 0.73 [95\%Cl 0. $48,1.10])$. Median overall survival was significantly improved for patients ages $65-74$ years treated with azacitidine compared with those who received CCR (14.2 versus 7.3 months, respectively; hazard ratio 0.64 [95\%Cl $0.42,0.97]$ ). Within the subgroup of patients preselected to low-dose cytarabine before randomization, median overall survival with azacitidine was 9.5 months versus 4.6 months with low-dose cytarabine (hazard ratio 0.77 [95\% $0.55,1.09]$ ). Within the low-dose cytarabine preselection group, patients with intermediate-risk cytogenetics who received azacitidine had a median overall survival of 14.1 months versus 6.4 months with low-dose cytarabine, and patients aged 65-74 years had median survival of 14.9 months versus 5.2 months, respectively. Overall response rates were similar with azacitidine and CCR (24.8\% and $17.3 \%$, respectively), but higher with azacitidine versus low-dose cytarabine (27.2\% and 13.9\%). Adverse events were generally comparable between the treatment arms.

Conclusions: Azacitidine may be the preferred treatment for patients with AML-MRC who are not candidates for intensive chemotherapy, particularly patients ages 65-74 years and those with intermediate-risk cytogenetics.

Trial registration: This study was registered at clinicalTrials.gov on February 16, 2010 (NCT01074047).

Keywords: Azacitidine, Low-dose cytarabine, Acute myeloid leukaemia, AML, Myelodysplasia-related changes, AMLMRC, Induction chemotherapy, Response, Survival

\section{Background}

Acute myeloid leukaemia (AML) is a heterogeneous disorder with multifactorial pathogenic mechanisms [1]. AML pathogenesis is characterised by recurrent chromosomal translocations and specific somatic mutations that define biologically distinct disease subtypes. The World Health Organization (WHO) classification of myeloid neoplasms includes four distinct types of AML [2]: AML with recurrent genetic abnormalities; therapyrelated AML (tAML); AML with myelodysplasia-related changes (AML-MRC); and AML not otherwise specified (AML-NOS). Among WHO-defined AML classifications, the most common are AML-MRC and AML-NOS [3]. AML-MRC is defined by the presence of multilineage dysplasia, prior history of myelodysplastic syndromes (MDS) (secondary AML [sAML]), or MDS-related cytogenetic abnormalities [4]. Compared with AML-NOS, patients with AML-MRC are generally older and more likely to have poor-risk cytogenetics, leading to poor response and prognosis [5]. Moreover, older patients with sAML tend to have disease that is more chemoresistant than do patients with AML-NOS [6]. While choice of AML treatment can depend on age, cytogenetic risk, performance status, and other factors, no particular therapy has yet been found to provide specific benefits in AML-MRC.

In the United States, azacitidine is recommended firstline therapy for patients with higher-risk MDS [7], and is indicated for all FAB subtypes of MDS and for treatment of low-blast-count AML (20-30\% bone marrow [BM] blasts) [8]. Azacitidine is also indicated in the European Union for higher-risk MDS and for treatment of adult patients with AML with any BM blast count who are unable to undergo allogeneic stem cell transplantation [9]. MDS and AML may reflect a continuum of myeloid disease, particularly in the older patient population with low white blood cell (WBC) counts. The international, randomised, phase 3 AZA-AML-001 (AZA-AML) study in older patients with newly diagnosed AML with $>30 \%$ BM blasts showed overall survival (OS) was 10.4 months in azacitidine-treated patients compared with 6.5 months for patients who received conventional care regimens $(C C R ; P=0.101)$ [10]. Consistent with azacitidine efficacy in higher-risk MDS, a prospective univariate analysis of 158 patients (32\% of all patients in the AZA-AML study) with locally diagnosed AML-MRC showed that azacitidine was associated with significantly improved OS compared with CCR $(P=0.036)$, and the difference approached statistical significance in multivariate analysis $(P=0.097)[10]$. Subsequently, central review of patients' BM samples identified a much larger proportion of patients who met WHO criteria for AML-MRC, suggesting the possibility of substantial under-diagnosis of AML-MRC in routine clinical practise. To help clarify whether patients with AML with myelodysplasia-related features respond preferentially to azacitidine, we evaluated efficacy and safety outcomes for this larger group of patients with centrally adjudicated AML-MRC in the AZA-AML trial.

\section{Methods}

The AZA-AML study was approved by the relevant institutional review boards or independent ethics 
committees and was conducted according to the Declaration of Helsinki. All patients provided written informed consent before study participation.

\section{Patients}

Inclusion and exclusion criteria and study design are reported in detail elsewhere [10]. Briefly, patients aged $\geq 65$ years with newly diagnosed de novo AML or sAML with $>30 \%$ BM blasts, Eastern Cooperative Oncology Group performance status (ECOG PS) scores 0-2, intermediate- or poor-risk cytogenetics per National Comprehensive Cancer Network (NCCN) 2009 criteria, and WBC counts $\leq 15 \times 10^{9} / \mathrm{L}$ were eligible. Before randomisation, patients were preselected to 1 of 3 protocolspecified CCR: intensive chemotherapy (IC; standard 7 +3 regimen with one induction course and up to two additional courses of consolidation), low-dose cytarabine (LDAC; $20 \mathrm{mg}$ BID for 10 days per 28-day treatment cycle), or best supportive care (BSC) only. Patients were then randomised $1: 1$ to azacitidine $\left(75 \mathrm{mg} / \mathrm{m}^{2} /\right.$ day for 7 days per 28-day cycle) or to CCR. Those randomised to CCR received their preselected treatment. All participants could receive BSC as needed.

Diagnosis and disease classification were recorded before study entry for each patient by the treating physician. Subsequently, assignment of AML-MRC according to WHO criteria was performed by the sponsor. Patients with centrally adjudicated AML-MRC must have met at least one of the following criteria: (1) dysplasia in $\geq 50 \%$ of cells in at least two of the three myeloid lineages, (2) sAML with antecedent history of MDS or myeloproliferative neoplasms (MPN), or (3) MDS-related cytogenetic abnormalities [4]. Dysplasia and cytogenetic assessments of baseline BM aspirate samples were performed centrally by an independent expert pathologist (John M. Bennett, MD) and cytogeneticist (Anne Hagemeijer, MD), blinded to local pathology and cytogenetic reports and treatment assignments. Proportions of dysplastic cells in the erythroid, granulocytic, and megakaryocytic lineages were determined by independent haematopathologist review of BM aspirates. History of MDS was based on local site reporting. Patients who met criteria for AML-MRC but who had received prior systemic anti-cancer therapy or prior radiation treatment were considered to have tAML per WHO definition and were excluded from analyses.

\section{Efficacy endpoints}

Analyses included only those patients in AZA-AML with a centrally confirmed AML-MRC diagnosis. The primary analysis compared the OS for azacitidine- and CCRtreated patients. Additionally, a sensitivity analysis of OS, similar to one that was predefined for the overall AZA-AML study [10], was performed, in which patients who had switched to alternate therapy at some point from their randomised treatment were censored at the time they did so.

Secondary analyses included Kaplan-Meier estimates of 1-year survival and overall response rate (i.e., complete remission $[\mathrm{CR}]$ plus $\mathrm{CR}$ with incomplete haematologic recovery [CRi]) as defined by International Working Group (IWG) response criteria for AML [11]. Stable disease was defined as the absence of an IWGdefined hematologic response with no evidence of disease progression, sustained for a period of $\geq 8$ weeks. Disease progression was defined as 1) $>50 \%$ increase in BM blast count percentage from baseline that persisted for $\geq 2 \mathrm{BM}$ assessments separated by $\geq 1$ month, unless the baseline count was $>70 \%$, in which case, $>70 \%$ blasts persisting for $2 \mathrm{BM}$ assessments separated by $\geq 1$ month; or 2) doubling of the baseline peripheral blood blast count that persisted for $\geq 7$ days with final peripheral blood blast count $>10 \times 10^{9} / \mathrm{L}$. OS outcomes with azacitidine and CCR were also evaluated within patient subgroups defined by cytogenetic risk (intermediate or poor) and by age (65-74 years or $\geq 75$ years).

Of all 488 patients enrolled in AZA-AML, 312 (64\%) were preselected to $\mathrm{LDAC}$ before randomisation to treatment; IC and BSC-only were each preselected for $18 \%$ of patients [10]. Because LDAC and azacitidine are both lower-intensity active therapies, OS and haematologic response analyses were also performed within the subgroup of patients with AML-MRC preselected to LDAC before randomisation, who then received azacitidine or LDAC on-study. Additionally, as IC was the other active CCR treatment option, OS and response were compared for patients preselected to IC who later received azacitidine or IC.

Exploratory analyses compared OS with azacitidine or CCR in patients who met only a single AML-MRC criterion (e.g., multilineage dysplasia but no history of prior MDS or MDS-related cytogenetics).

\section{Safety}

The safety population included all patients who received at least 1 dose of study drug (or at randomisation for the BSC-only group) and had at least 1 safety assessment thereafter. Treatment-emergent adverse events (TEAEs) were defined as new or worsening AEs between the time of first dose (or at randomisation for BSC-only patients) until 28 days after the last dose of azacitidine or LDAC, 70 days after the last dose of IC, or the day of discontinuation and/or study closure for patients receiving BSC only. TEAEs were coded by Medical Dictionary for Regulatory Activities (MedDRA), and graded for severity using the National Cancer Institute Common Toxicity Criteria for Adverse Events (NCI-CTCAE) version 4.0. 


\section{Statistical methods}

Demographic and disease characteristics at baseline are reported descriptively. Median OS and 1-year survival rates were determined based on Kaplan-Meier product limit estimates. Hazard ratios (HR) and 95\% CI for OS are estimated from unstratified Cox proportional hazards models. As these OS analyses are post hoc in nature, multiplicity of testing and power considerations did not allow for a proper interpretation of $P$ values; therefore, results are presented as point estimates with corresponding 95\% confidence intervals (CI). To assess heterogeneity, 95\% CI for OS within individual CCR arms (IC, LDAC, and BSC) were constructed. Overall response rate was compared between azacitidine and CCR using summary statistics.

\section{Results}

\section{Baseline demographic and disease characteristics}

In all, 262 patients (54\% of all patients in AZA-AML) fulfilled WHO criteria for AML-MRC upon central review; 129 were treated with azacitidine and 133 received CCR (IC $n=24$, LDAC $n=79$, BSC only $n=30$ ). This AML-MRC subgroup represents $53 \%$ of all azacitidinetreated patients and $54 \%$ of all CCR patients in the AZA-AML study. Interestingly, of all patients in the
CCR arm who received BSC only in the AZA-AML study, 67\% (30/45) had AML-MRC, suggesting AMLMRC patients were frequently considered to be at higher-risk by their managing physicians during treatment preselection.

In both treatment arms, median ages were greater than 75 years, and approximately one-half of all patients had poor-risk cytogenetics (Table 1). Ninety-three patients (35\%) met two or more AML-MRC criteria, including 15 patients (6\%) who met all three criteria (Fig. 1). Of 87 patients identified locally as having a prior diagnosis of MDS before study entry, 8 were not classified as AML-MRC in the central review because they met at least one WHO-defined exclusion criterion (e.g., prior systemic anti-cancer treatment). In all, among 262 patients with centrally defined AML-MRC, 79 (30\%) had had prior MDS, 138 (53\%) had MDS-related cytogenetic abnormalities, and 153 (58\%) had morphologic multilineage dysplasia. Notably, of the 153 patients determined to have multilineage dysplasia based on central review of BM aspirates, 89 patients were originally reported to have had no or only one dysplastic lineage according to local assessment, and 66 of these 89 patients (74\%) were not classified as having AML-MRC at local diagnosis.

Table 1 Baseline demographic and disease characteristics

\begin{tabular}{|c|c|c|c|c|}
\hline & \multicolumn{2}{|c|}{ All patients with AML-MRC $(N=262)$} & \multicolumn{2}{|c|}{ LDAC-preselected patients with AML-MRC $(n=160)$} \\
\hline & Azacitidine $(n=129)$ & $\operatorname{CCR}(n=133)$ & Azacitidine $(n=81)$ & $\operatorname{LDAC}(n=79)$ \\
\hline Age (years), median (min, max) & $76(64,90)$ & $75(65,87)$ & $76(64,90)$ & $75(65,87)$ \\
\hline Age $\geq 75$ years, $n(\%)$ & $77(60)$ & $69(52)$ & $55(68)$ & $41(52)$ \\
\hline Male gender, $n \%$ & $81(63)$ & $78(59)$ & $42(52)$ & $43(54)$ \\
\hline Prior history of MDS*, $n(\%)$ & $44(34)$ & $35(26)$ & $32(40)$ & $20(25)$ \\
\hline \multicolumn{5}{|l|}{ ECOG PS, $n(\%)$} \\
\hline $0-1$ & $94(73)$ & $104(78)$ & $56(69)$ & $64(81)$ \\
\hline 2 & $35(27)$ & $29(22)$ & $25(31)$ & $15(19)$ \\
\hline \multicolumn{5}{|l|}{ Cytogenetic risk, n (\%) } \\
\hline Intermediate & $63(49)$ & $61(46)$ & $47(58)$ & $33(42)$ \\
\hline Poor & $66(51)$ & $72(54)$ & $34(42)$ & $46(58)$ \\
\hline \multicolumn{5}{|l|}{ No. of dysplastic lineages, $n(\%)$} \\
\hline $0-1$ & $57(44)$ & $52(39)$ & $36(44)$ & $35(44)$ \\
\hline $2-3$ & $72(56)$ & $81(61)$ & $45(56)$ & $44(56)$ \\
\hline \% BM blasts, median (min, max) & $65.0(27,99)$ & $70.0(26,100)$ & $66.0(27,99)$ & $69.0(31,100)$ \\
\hline \multicolumn{5}{|l|}{ Haematology, median (min, max) } \\
\hline $\operatorname{ANC}\left(10^{9} / \mathrm{L}\right)$ & $0.4(0.0,11.6)$ & $0.3(0.0,8.7)$ & $0.4(0.0,11.6)$ & $0.3(0.0,8.7)$ \\
\hline Platelets $\left(10^{9} / \mathrm{L}\right)$ & $56(3,585)$ & $55(6,244)$ & $58(7,585)$ & $55(6,244)$ \\
\hline WBC $\left(10^{9} / \mathrm{L}\right)$ & $3.2(0.6,26.5)$ & $2.4(0.4,22.6)$ & $2.6(0.6,26.5)$ & $2.3(0.4,13.5)$ \\
\hline $\mathrm{Hgb}(\mathrm{g} / \mathrm{dL})$ & $9.5(5.0,13.4)$ & $9.3(5.0,14.4)$ & $9.4(5.0,11.8)$ & $9.4(5.6,14.4)$ \\
\hline
\end{tabular}

*Based on local site reporting

AML-MRC, AML with myelodysplasia-related changes; ANC, absolute neutrophil count; BM, bone marrow; CCR, conventional care regimens; ECOG PS, Eastern Cooperative Oncology Group performance status; Hgb, haemoglobin; LDAC, low-dose cytarabine; MDS, myelodysplastic syndromes; WBC, white blood cell count 


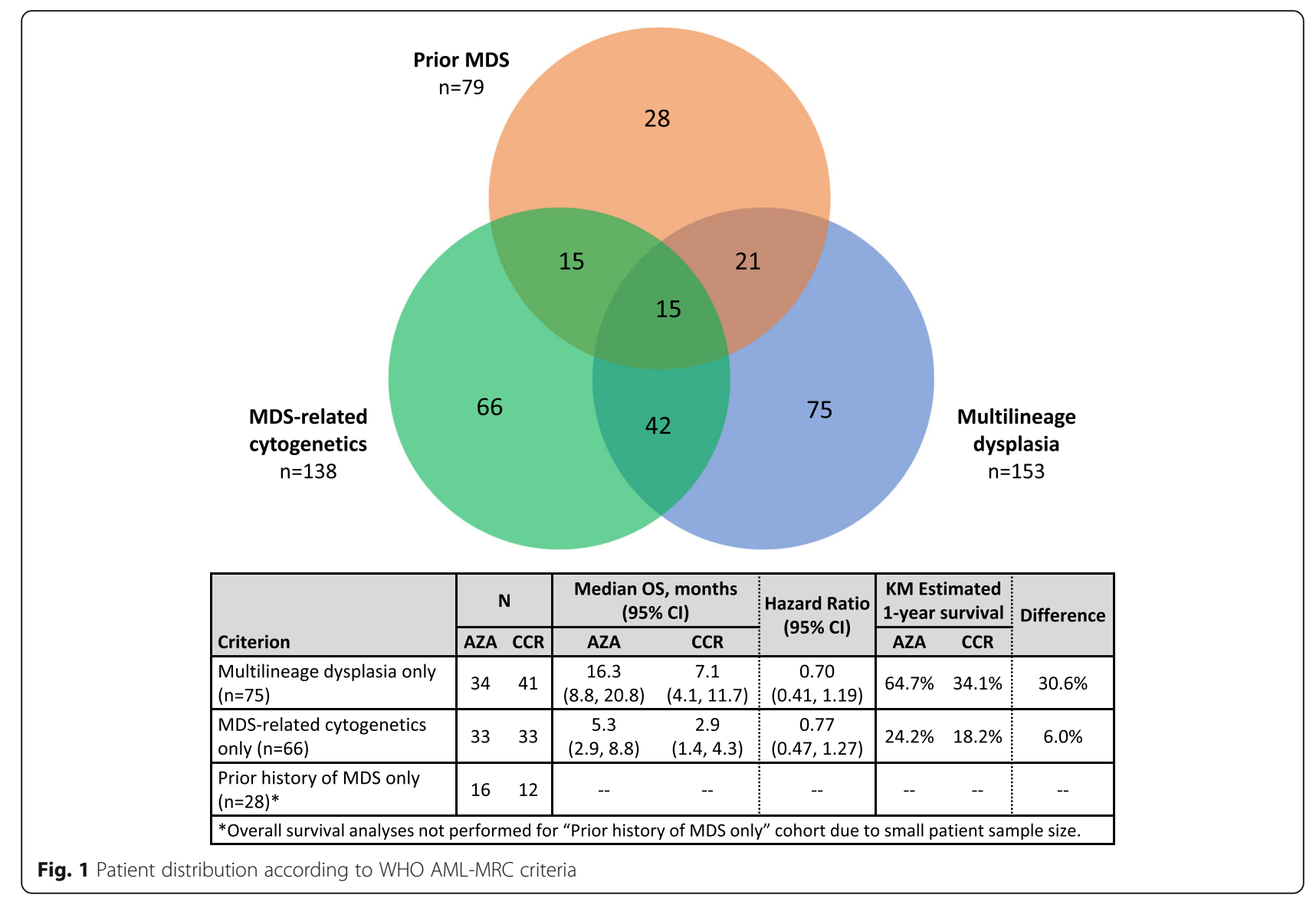

The majority of the AML-MRC population (160/262; $61 \%)$ had been preselected to LDAC before study randomisation. Baseline characteristics of LDACpreselected patients who ultimately received azacitidine $(n=81)$ or LDAC $(n=79)$ were generally comparable (Table 1), although proportionately more azacitidinetreated patients had an ECOG PS score of $2(31 \%$ vs $19 \%$ of patients treated with LDAC) and proportionately more LDAC-treated patients had poor-risk cytogenetics (58\% vs $42 \%$ of patients treated with azacitidine). Azacitidine-treated patients also tended to be older; $68 \%$ of patients in this preselection group were aged $\geq 75$ years, compared with $52 \%$ of LDAC-treated patients.

Patients in the azacitidine arm received a median of five treatment cycles (range 1-27), and those in the CCR arm received a median of two IC treatment cycles (1-3), two LDAC treatment cycles (1-22), or three 28-day cycles of BSC-only (1-9). For patients preselected to receive LDAC but treated with azacitidine, the median number of azacitidine treatment cycles was six (range 1-25).

\section{Survival: All AML-MRC patients}

Median OS among all patients with AML-MRC was significantly prolonged in the azacitidine arm compared with the CCR arm (8.9 vs 4.9 months, respectively; HR 0.74, 95\%CI 0.57, 0.97) (Table 2; Fig. 2a), and the estimated 1-year survival rate was $17.4 \%$ greater in the azacitidine arm (44.3\% vs $27.2 \%$ with CCR). In the survival sensitivity analysis, in which patients were censored at the time they received alternate therapy, median OS increased in both groups, to 11.4 months for azacitidinetreated patients and to 5.4 months for patients who received CCR (HR 0.72, 95\%CI 0.52, 1.0); 1-year survival estimates were $49.4 \%$ vs. $31.0 \%$, respectively (Fig. $2 \mathrm{~b})$. There was no evidence of significant heterogeneity of treatment effect among the $3 \mathrm{CCR}$ treatments; median OS in the IC, LDAC, and BSC-only groups were 8.9 months $(95 \% \mathrm{CI} 3.2,15.1), 4.6$ months $(3.3,6.4)$, and 3.8 months $(2.0,8.0)$, respectively. Within the subgroup of patients preselected to IC, median OS in ICpreselected patients who received azacitidine $(n=22)$ was slightly improved at 11.6 months compared with 8.9 months among IC-treated patients $(n=24)$, but the difference was not statistically significant (HR 0.83, 95\% CI 0.42, 1.62).

Though the subgroup of patients with prior MDS as their sole AML-MRC criterion was too small for meaningful comparisons $(n=28)$, patients who only met the AML-MRC criterion of morphologic multilineage 
Table 2 Survival outcomes among all patients with AML-MRC $(N=262)$ and among those preselected to LDAC $(n=160)$

\begin{tabular}{|c|c|c|c|c|c|c|c|c|c|}
\hline \multicolumn{10}{|c|}{ All patients with AML-MRC $(\mathrm{N}=262)$} \\
\hline & \multicolumn{2}{|l|}{$N$} & \multicolumn{2}{|c|}{ Median OS, months $(95 \% \mathrm{Cl})$} & \multirow[t]{2}{*}{ Difference, months } & \multirow[t]{2}{*}{ Hazard ratio $(95 \% \mathrm{Cl})$} & \multicolumn{2}{|c|}{ K-M Estimated 1-Year survival } & \multirow[t]{2}{*}{ Difference } \\
\hline & AZA & CCR & AZA & CCR & & & AZA & CCR & \\
\hline Overall & 129 & 133 & $8.9(6.9,12.9)$ & $4.9(3.8,6.5)$ & 4.0 & $0.74(0.57,0.97)$ & $44.3 \%$ & $26.9 \%$ & $17.4 \%$ \\
\hline \multicolumn{10}{|c|}{ Cytogenetic risk } \\
\hline Intermediate & 63 & 61 & $16.4(12.9,19.7)$ & $8.9(5.4,13.7)$ & 7.5 & $0.73(0.48,1.10)$ & $65.1 \%$ & $42.4 \%$ & $22.7 \%$ \\
\hline Poor & 66 & 72 & $5.0(3.6,7.2)$ & $3.2(2.2,4.7)$ & 1.8 & $0.79(0.55,1.11)$ & $23.9 \%$ & $13.7 \%$ & $10.2 \%$ \\
\hline \multicolumn{10}{|l|}{ Age } \\
\hline $65-74$ years & 52 & 64 & $14.2(10.8,18.7)$ & $7.3(4.8,11.3)$ & 6.9 & $0.64(0.42,0.97)$ & $59.6 \%$ & $32.5 \%$ & $27.2 \%$ \\
\hline$\geq 75$ years & 77 & 69 & $5.9(4.5,9.2)$ & $3.8(2.6,5.1)$ & 2.1 & $0.77(0.54,1.09)$ & $33.8 \%$ & $21.4 \%$ & $12.3 \%$ \\
\hline \multicolumn{10}{|c|}{ LDAC-preselected patients with AML-MRC $(n=160)$} \\
\hline & \multicolumn{2}{|l|}{ N } & \multicolumn{2}{|c|}{ Median OS, months (95\%Cl) } & \multirow[t]{2}{*}{ Difference, months } & \multirow[t]{2}{*}{ Hazard ratio $(95 \% \mathrm{Cl})$} & \multicolumn{2}{|c|}{ K-M Estimated 1-Year survival } & Difference \\
\hline & AZA & LDAC & AZA & LDAC & & & AZA & LDAC & \\
\hline Overall & 81 & 79 & $9.5(5.9,14.1)$ & $4.6(3.3,6.4)$ & 4.9 & $0.77(0.55,1.09)$ & $45.3 \%$ & $23.5 \%$ & $21.8 \%$ \\
\hline \multicolumn{10}{|c|}{ Cytogenetic risk } \\
\hline Intermediate & 47 & 33 & $14.1(8.9,17.6)$ & $6.4(3.8,14.2)$ & 7.7 & $0.90(0.54,1.50)$ & $57.4 \%$ & $34.4 \%$ & $23.0 \%$ \\
\hline Poor & 34 & 46 & $5.6(2.2,9.5)$ & $3.7(2.2,5.1)$ & 1.9 & $0.83(0.52,1.33)$ & $28.1 \%$ & $15.8 \%$ & $12.3 \%$ \\
\hline \multicolumn{10}{|l|}{ Age } \\
\hline $65-74$ years & 26 & 38 & $14.9(9.0,19.6)$ & $5.2(3.5,10.0)$ & 9.7 & $0.68(0.39,1.18)$ & $61.5 \%$ & $26.3 \%$ & $35.2 \%$ \\
\hline$\geq 75$ years & 55 & 41 & $8.8(4.5,12.9)$ & $4.0(2.8,6.4)$ & 4.8 & $0.78(0.50,1.22)$ & $37.5 \%$ & $20.8 \%$ & $16.7 \%$ \\
\hline
\end{tabular}

AML-MRC, AML with myelodysplasia-related changes; AZA, azacitidine; CCR, conventional care regimens; Cl, confidence interval; K-M, Kaplan-Meier Hazard ratios in bold indicate statistical significance

dysplasia ( $n=75$ : azacitidine $n=34$; CCR $n=41)$ had better OS outcomes than the group of all AML-MRC patients: median OS with azacitidine was 16.3 months vs 7.1 months with CCR (HR 0.70, 95\%CI 0.41, 1.2), and estimated 1-year survival rates were $64.7 \%$ vs $34.1 \%$, respectively. In contrast, patients whose only AML-MRC feature was MDS-related cytogenetics (AZA $n=33$, CCR $n=33$ ) fared much worse than overall: median OS with azacitidine vs CCR was 5.3 vs 2.9 months, respectively (HR $0.77,95 \% \mathrm{CI} 0.47$, 1.3 ), and 1 -year survival was $24.2 \%$ vs $18.2 \%$.

Overall, 124 patients (47\%) had intermediate-risk cytogenetics and 138 patients (53\%) had poor-risk cytogenetics. For patients with intermediate-risk cytogenetics treated with azacitidine, median OS was 16.4 months compared with 8.9 months among patients treated with CCR (HR 0.73, 95\%CI 0.48, 1.1) (Fig. 2c), with estimated 1-year survival rates of $65.1 \%$ and $42.4 \%$, respectively (Table 2). As expected, poorrisk cytogenetics was associated with substantially worse prognosis; median OS with azacitidine was 5.0 months compared with 3.2 months with CCR (HR $0.79,95 \% \mathrm{CI} 0.55,1.1)$ and estimated 1-year survival rates were $23.9 \%$ and $13.7 \%$, respectively (Table 2 and Fig. 2c).
Overall, 116 patients were aged $65-74$ years and 146 patients were aged $\geq 75$ years (azacitidine age range 75-90 years; CCR age range $75-87$ years). In the younger cohort, median OS among patients treated with azacitidine $(n=52)$ was significantly prolonged compared with that for CCR-treated patients ( $n=64$ : LDAC $n=38$, IC $n=18$; BSC only $n=8)$ : 14.2 vs 7.3 months, respectively (HR $0.64,95 \% \mathrm{CI}$ $0.42,0.97$ ) (Fig. 2d), with estimated 1-year survival rates of $59.6 \%$ for patients treated with azacitidine and $32.5 \%$ for those who received CCR (Table 2). Among those aged $\geq 75$ years, median OS was 5.9 months with azacitidine $(n=77)$ and 3.8 months with CCR ( $n=69$ : LDAC $n=41$; IC $\mathrm{n}=6$; BSC only $n=22)($ HR $0.77,95 \%$ CI 0.54, 1.09) (Table 2).

\section{Survival: LDAC preselection subgroup}

Median OS among LDAC-preselected patients treated with azacitidine was double that for LDAC-treated patients (9.5 vs 4.6 months, respectively; HR $0.77,95 \% \mathrm{CI}$ $0.55,1.09$ ) (Fig. 3a), and estimated 1-year survival rates were $45.3 \%$ vs $23.5 \%$, respectively (Table 2 ).

Equal numbers of patients preselected to receive LDAC had NCCN-defined intermediate-risk cytogenetics $(n=80$; 

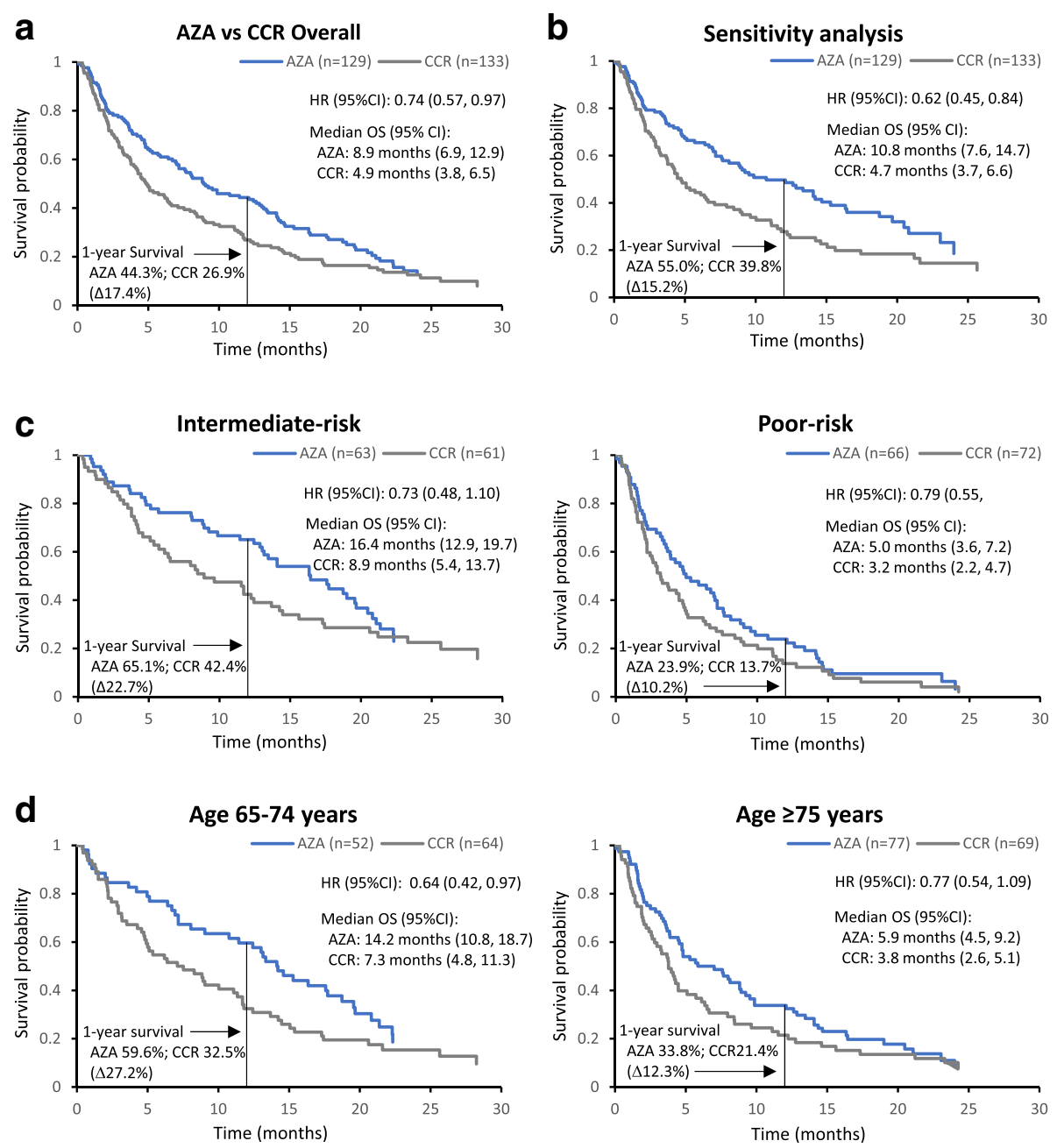

Fig. 2 Overall survival among patients with AML-MRC. a Overall survival for all patients with AML-MRC treated with azacitidine or CCR. b Sensitivity analysis, in which patients who switched to alternate therapy were censored at the time they did so. c Overall survival by NCCN cytogenetic risk. $\mathbf{d}$ Overall survival by age group

azacitidine $n=47$, LDAC $n=33$ ) and poor-risk cytogenetics $(\mathrm{n}=80$; azacitidine $n=34$, LDAC $n=46)$. Among patients with intermediate-risk cytogenetics, azacitidine treatment was associated with a 7.7-month improvement in median OS compared with LDAC: 14.1 vs 6.4 months, respectively (HR $0.90,95 \% \mathrm{CI} 0.54,1.50$ ) (Fig. $3 \mathrm{~b}$ and Table 2). For patients with poor-risk cytogenetics, median OS with azacitidine vs. LDAC was 5.6 vs. 3.7 months, respectively (HR 0.83, 95\%CI 0.52, 1.33) (Table 2).

Patients aged 65-74 years treated with azacitidine $(n=26)$ fared better than those treated with LDAC $(\mathrm{n}=$ 38), with a 9.7-month improvement in median OS: 14.9 vs 5.2 months, respectively (HR $0.68,95 \%$ CI $0.39,1.18$ ) (Fig. $3 \mathrm{c}$ and Table 2 ). Among patients aged $\geq 75$ years ( $n$ $=55$ ), median OS was more than doubled in azacitidinetreated patients compared with LDAC-treated patients $(n=41)$ at 8.8 vs. 4.0 months, respectively (HR 0.78 , 95\%CI 0.50, 1.22) (Table 2).

\section{Response}

Overall response rates (i.e., proportion of patients with $\mathrm{CR}$ or $\mathrm{CRi}$ ) were $24.8 \%$ and $17.3 \%$ with azacitidine and CCR, respectively. Despite the small improvement in median OS among patients with AMLMRC preselected to receive IC who received azacitidine, the overall response rate with azacitidine in the IC-preselection subgroup was only $27 \%$, vs. $50 \%$ for patients who received IC. Response rates were slightly higher with azacitidine than with CCR in both cytogenetic risk groups, and response rates were higher within each treatment arm for patients with intermediate-risk cytogenetics compared with poorrisk cytogenetics (Table 3). Among patients aged $\geq 75$ years, overall response rate was greater in the azacitidine arm (22.1\% vs $10.1 \%$ with CCR).

Patients preselected to receive LDAC who were treated with azacitidine were more likely to have had a 

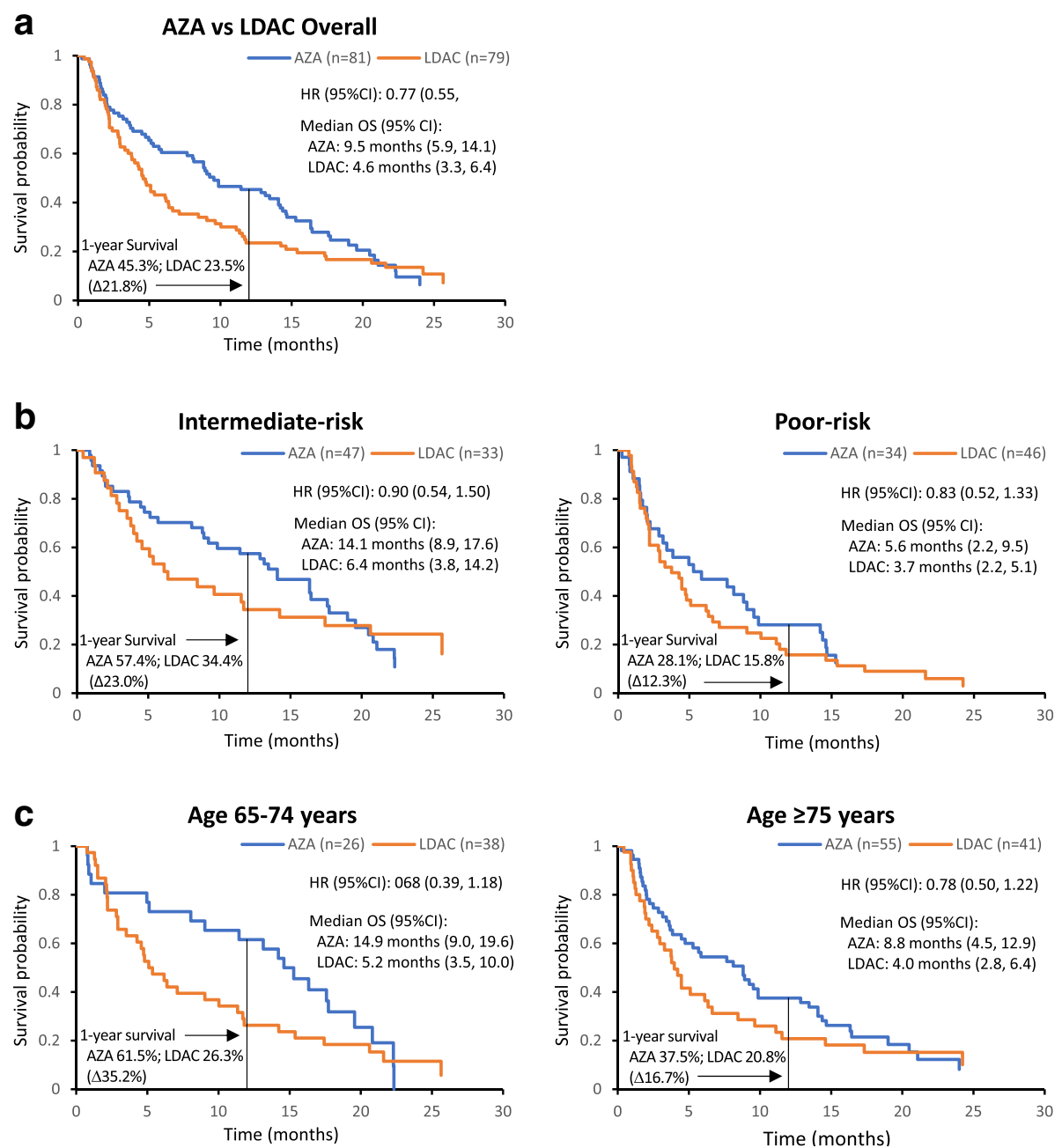

Fig. 3 Overall survival among patients with AML-MRC preselected to LDAC. a Overall survival for patients with AML-MRC treated with azacitidine or LDAC; $\mathbf{b}$ Overall survival by cytogenetic risk; c Overall survival by age group

CR or CRi $(27.2 \%$ vs. $13.9 \%$ with LDAC) and LDACtreated patients were more likely to have progressive disease (13.9\% vs. $4.9 \%$ with azacitidine) (Table 4$)$. Interestingly, no patient with poor-risk cytogenetics in the azacitidine arm had progressive disease as their best response, compared with 9 of 46 patients (19.6\%) treated with LDAC.

\section{Safety}

The safety-evaluable cohort comprised 258 patients (99\%). Grade 3-4 TEAEs were reported for $87 \%$ of patients in each treatment arm, and incidences of individual TEAEs were generally comparable between the azacitidine and CCR arms (Table 5). Rates of grade 3-4 haematological TEAEs decreased over time during continued treatment in both treatment arms
(Table 6). TEAEs in patients with AML-MRC were generally consistent with those reported for all patients in AZA-AML [10].

\section{Discussion}

While slightly less than one-third of patients in the AZA-AML study were identified as having AMLMRC at local diagnosis, more than one-half of all patients were so identified upon central review. The discrepancy between local and central adjudication suggests that diagnosing dysplasia in routine clinical practice is a challenge, although dysplasia assessments are known to be subject to high interobserver variability [12]. As noted, 89 patients in AZA-AML with multilineage dysplasia determined in independent central review of $\mathrm{BM}$ aspirates were originally reported to have single-lineage or no dysplasia in local 
Table 3 Response in patients with AML-MRC treated with azacitidine or CCR

\begin{tabular}{|c|c|c|c|c|}
\hline \multicolumn{5}{|c|}{ All patients with AML-MRC $(N=262)$} \\
\hline & \multicolumn{2}{|l|}{$\operatorname{AZA}(n=129)$} & \multicolumn{2}{|l|}{$\operatorname{CCR}(n=133)$} \\
\hline & \multicolumn{2}{|l|}{$n(\%)$} & & \\
\hline Overall Response Rate (CR + CRi) & \multicolumn{2}{|l|}{$32(24.8)$} & \multicolumn{2}{|l|}{$23(17.3)$} \\
\hline$C R$ & \multicolumn{2}{|l|}{$25(19.4)$} & \multicolumn{2}{|l|}{$20(15.0)$} \\
\hline CRi & \multicolumn{2}{|l|}{$7(5.4)$} & \multicolumn{2}{|l|}{$3(2.3)$} \\
\hline Partial remission & \multicolumn{2}{|l|}{$1(0.8)$} & \multicolumn{2}{|l|}{$2(1.5)$} \\
\hline Stable disease & \multicolumn{2}{|l|}{$46(35.7)$} & \multicolumn{2}{|l|}{$38(28.6)$} \\
\hline \multirow[t]{4}{*}{ Progressive disease } & \multicolumn{2}{|l|}{$11(8.5)$} & \multicolumn{2}{|l|}{$16(12.0)$} \\
\hline & \multicolumn{2}{|c|}{ AML-MRC patients with Intermediate-risk cytogenetics } & \multicolumn{2}{|c|}{ AML-MRC patients with Poor-risk cytogenetics } \\
\hline & $\mathrm{AZA}(n=63)$ & $\operatorname{CCR}(n=61)$ & $\mathrm{AZA}(n=66)$ & $\operatorname{CCR}(n=72)$ \\
\hline & \multicolumn{4}{|l|}{ n (\%) } \\
\hline Overall Response Rate (CR + CRi) & $21(33.3)$ & $15(24.6)$ & $11(16.7)$ & $8(11.1)$ \\
\hline$C R$ & $18(28.6)$ & $13(21.3)$ & $7(10.6)$ & $7(9.7)$ \\
\hline CRi & $3(4.8)$ & $2(3.3)$ & $4(6.1)$ & $1(1.4)$ \\
\hline Partial remission & $1(1.6)$ & $1(1.6)$ & 0 & $1(1.4)$ \\
\hline Stable disease & $25(39.7)$ & $18(29.5)$ & $21(31.8)$ & $20(27.8)$ \\
\hline \multirow[t]{4}{*}{ Progressive disease } & $6(9.5)$ & $5(8.2)$ & $5(7.6)$ & $11(15.3)$ \\
\hline & \multicolumn{2}{|c|}{ AML-MRC patients ages $65-74$ years } & \multicolumn{2}{|c|}{ AML-MRC patients ages $\geq 75$ years } \\
\hline & $\mathrm{AZA}(n=52)$ & $\operatorname{CCR}(n=64)$ & $\mathrm{AZA}(n=77)$ & $\operatorname{CCR}(n=69)$ \\
\hline & \multicolumn{4}{|l|}{ n (\%) } \\
\hline Overall Response Rate (CR + CRi) & $15(28.8)$ & $16(25.0)$ & $17(22.1)$ & $7(10.1)$ \\
\hline$C R$ & $11(21.2)$ & $14(21.9)$ & $14(18.2)$ & $6(8.7)$ \\
\hline CRi & $4(7.7)$ & $2(3.1)$ & $3(3.9)$ & $1(1.4)$ \\
\hline Partial remission & 0 & $2(3.1)$ & $1(1.3)$ & 0 \\
\hline Stable disease & $20(38.5)$ & $24(37.5)$ & $26(33.8)$ & $14(20.3)$ \\
\hline Progressive disease & $5(9.6)$ & $6(9.4)$ & $6(7.8)$ & $10(14.5)$ \\
\hline
\end{tabular}

AML-MRC, AML with myelodysplasia-related changes; AZA, azacitidine; CCR, conventional care regimens; CR, complete remission; CRi, CR with incomplete blood count recovery

assessments, and 66 of these patients were not considered to have had AML-MRC at study entry. It is also noteworthy that only $\sim 30 \%$ of centrally-defined AML-MRC cases were associated with locally reported prior MDS; a potential limitation of the current analysis was the inability to independently verify local reports of prior MDS. Potential underrecognition of prior MDS is consistent with results of a recent study indicating that the leukaemia cells of some older patients with apparent de novo AML harboured MDS-associated mutations, suggesting that such leukaemias may have had an unrecognised MDS prodrome [13].

The prognostic significance of morphologic dysplasia in AML has been analysed extensively. A preponderance of evidence suggests that dysplasia alone has no overall prognostic value $[3,14,15]$, although within the AML-MRC classification, the presence of $>50 \%$ micromegakaryocytes or $>50 \%$ hypogranulated myeloid cells has been associated with poorer eventfree survival [16]. Prognosis in AML-MRC may be better defined by the associated mutational profile; for example, in the presence of wild-type rather than mutated NPM1 [17], or the presence of mutated ASXL1 or TP53 [18]. In the current analysis, patients with multilineage dysplasia only (i.e., no prior MDS or MDS-related cytogenetics) treated with azacitidine had much improved OS (16.3 months) compared with all AML-MRC azacitidine-treated patients (8.9 months), and compared with similar CCRtreated patients (7.1 months), suggesting that the presence of multilineage dysplasia alone may have prognostic value. Accurate morphologic assessment of multilineage dysplasia may be important for 
Table 4 Response among patients with AML-MRC preselected to receive LDAC and treated with azacitidine or LDAC

\begin{tabular}{|c|c|c|c|c|}
\hline \multicolumn{5}{|c|}{ Patients with AML-MRC preselected to receive LDAC $(n=160)$} \\
\hline & \multicolumn{2}{|l|}{ AZA $(n=81)$} & \multicolumn{2}{|l|}{$\operatorname{LDAC}(n=79)$} \\
\hline & \multicolumn{2}{|l|}{$n(\%)$} & & \\
\hline Overall Response Rate (CR + CRi) & \multicolumn{2}{|l|}{$22(27.2)$} & \multicolumn{2}{|l|}{$11(13.9)$} \\
\hline$C R$ & \multicolumn{2}{|l|}{$17(21.0)$} & \multicolumn{2}{|l|}{$10(12.7)$} \\
\hline CRi & \multicolumn{2}{|l|}{$5(6.2)$} & \multicolumn{2}{|l|}{$1(1.3)$} \\
\hline Partial remission & \multicolumn{2}{|l|}{$1(1.2)$} & \multicolumn{2}{|l|}{$1(1.3)$} \\
\hline Stable disease & \multicolumn{2}{|l|}{$30(37.0)$} & \multicolumn{2}{|l|}{$30(38.0)$} \\
\hline \multirow[t]{4}{*}{ Progressive disease } & $4(4.9)$ & & \multicolumn{2}{|l|}{$11(13.9)$} \\
\hline & \multicolumn{2}{|c|}{ LDAC-preselected Intermediate-risk cytogenetics } & \multicolumn{2}{|c|}{ LDAC-preselected Poor-risk cytogenetics } \\
\hline & AZA $(n=47)$ & $\operatorname{LDAC}(n=33)$ & $\mathrm{AZA}(n=34)$ & $\operatorname{LDAC}(n=46)$ \\
\hline & \multicolumn{4}{|l|}{$n(\%)$} \\
\hline Overall Response Rate (CR + CRi) & $15(31.9)$ & $6(18.2)$ & $7(20.6)$ & $5(10.9)$ \\
\hline$C R$ & $12(25.5)$ & $6(18.2)$ & $5(14.7)$ & $4(8.7)$ \\
\hline CRi & $3(6.4)$ & 0 & $2(5.9)$ & $1(2.2)$ \\
\hline Partial remission & $1(2.1)$ & $1(3)$ & 0 & 0 \\
\hline Stable disease & $19(40.4)$ & $15(45.5)$ & $11(32.4)$ & $15(32.6)$ \\
\hline \multirow[t]{4}{*}{ Progressive disease } & $4(8.5)$ & $2(6.1)$ & 0 & $9(19.6)$ \\
\hline & \multicolumn{2}{|c|}{ LDAC-preselected age $65-74$ years } & \multicolumn{2}{|c|}{ LDAC-preselected age $\geq 75$ years } \\
\hline & AZA $(n=26)$ & $\operatorname{LDAC}(n=38)$ & $\mathrm{AZA}(n=55)$ & $\operatorname{LDAC}(n=41)$ \\
\hline & \multicolumn{2}{|l|}{$n(\%)$} & & \\
\hline Overall Response Rate (CR + CRi) & $10(38.5)$ & $7(18.4)$ & $12(21.8)$ & $4(9.8)$ \\
\hline$C R$ & $7(26.9)$ & $6(15.8)$ & $10(18.2)$ & $4(9.8)$ \\
\hline CRi & $3(11.5)$ & $1(2.6)$ & $2(3.6)$ & 0 \\
\hline Partial remission & 0 & $1(2.6)$ & $1(1.8)$ & 0 \\
\hline Stable disease & $9(34.6)$ & $18(47.4)$ & $21(38.2)$ & $12(29.3)$ \\
\hline Progressive disease & $2(7.7)$ & $4(10.5)$ & $2(3.6)$ & $7(17.1)$ \\
\hline
\end{tabular}

AML-MRC, AML with myelodysplasia-related changes; $A Z A$, azacitidine; $C R$, complete remission; $C R i, C R$ with incomplete blood count recovery; LDAC, low-dose cytarabine

identifying the patients who might benefit most from azacitidine.

Even in this study of older patients with AML, the AML-MRC subgroup presented substantial treatment challenges; $57 \%$ of these patients were aged $\geq 75$ years, $53 \%$ had poor-risk cytogenetics, and approximately one-third were reported to have had antecedent MDS. In the overall AZA-AML study population, only $\sim 35 \%$ of patients had poor-risk cytogenetics, and as expected, proportionately fewer patients had antecedent MDS/MPN ( 20\%) [10]. Consistent with this prognostic profile, the median OS for AZA-AML patients treated with azacitidine was 8.9 months for patients with AML-MRC, compared with 10.4 months for all azacitidine-treated patients in the study. Likewise, median OS with CCR was 4.9 months for patients with AML-MRC compared with 6.5 months among all CCR-treated patients. Nevertheless, the relative survival benefit for azacitidine observed overall in the study was also maintained in the AMLMRC population, with a significant improvement in median OS of 4.0 months and a $26 \%$ reduced risk of death compared with CCR. Additionally, median OS with azacitidine was almost double that of CCR in patients with intermediate-risk cytogenetics and among "younger" patients (ages 65-74 years) compared with CCR.

This analysis also suggested an OS benefit with azacitidine in the subgroup of patients preselected to receive low-intensity LDAC treatment before randomisation. Median OS with azacitidine was more than twice that with LDAC in patients with AMLMRC. Keeping in mind that comparisons between azacitidine and LDAC by age and cytogenetic risk 
Table 5 Most frequent ( $\geq 5 \%$ of patients) grade 3-4 treatmentemergent adverse events (TEAEs)

\begin{tabular}{lll}
\hline Preferred term & Azacitidine $(n=128) n(\%)$ & CCR $(n=130) n(\%)$ \\
\hline Any grade 3-4 TEAE & $111(87)$ & $113(87)$ \\
Thrombocytopenia & $33(26)$ & $27(21)$ \\
Febrile neutropenia & $29(23)$ & $43(33)$ \\
Neutropenia & $28(22)$ & $25(19)$ \\
AML $^{*}$ & $25(20)$ & $23(18)$ \\
Pneumonia & $24(19)$ & $18(14)$ \\
Anaemia & $19(15)$ & $21(16)$ \\
Pyrexia & $13(10)$ & $9(7)$ \\
Hypokalaemia & $9(7)$ & $10(8)$ \\
Leukopenia & $8(6)$ & $10(8)$ \\
Sepsis & $7(6)$ & $9(7)$ \\
Decreased appetite & $6(5)$ & $2(2)$ \\
Dyspnoea & $6(5)$ & $4(3)$ \\
\hline
\end{tabular}

Safety-evaluable patients received at least 1 dose of study drug and had at least 1 post-baseline safety assessment. Patients who received BSC only were included in safety assessments if they had at least 1 postrandomisation safety assessment

*Worsening disease

comprised relatively small patient subgroups, median OS was more than twice as long for LDACpreselected patients with intermediate-risk cytogenetics treated with azacitidine, and almost 3-fold longer for patients aged 65-74 years. While they did not fare as well, azacitidine-treated patients with poor-risk cytogenetics, and those aged $\geq 75$ years, had 2 - to 5 month improvement in median OS compared with LDAC-treated patients. LDAC has previously been shown to be ineffective in patients with AML with adverse cytogenetics [19]. It should be noted that better outcomes for azacitidine-treated patients in this preselection group may in part reflect differences in treatment exposure; the median number of azacitidine treatment cycles for this population was 3 times that of LDAC treatment cycles (6 vs 2 cycles). Nevertheless, compared with all LDACtreated patients in AZA-AML $(n=158)$, LDAC-treated patients with AML-MRC $(n=79,50 \%)$ had a somewhat lower median OS (6.4 vs 4.6 months, respectively), suggesting that LDAC may not be optimal in this patient population. Another approach that has shown promise in a partially overlapping patient population to that evaluated in this study is CPX-351 (a liposomal formulation of cytarabine and daunorubicin at a fixed 5:1 $\mathrm{M}$ ratio) which has been compared in a phase 3 study to standard " $7+3$ " induction chemotherapy in 309 patients aged 60-75 years with previously untreated sAML, tAML, or AML with MDS-related cytogenetic abnormalities [20]. CPX-351 treatment resulted in a median OS of 9.6 months compared with 6 months with standard induction chemotherapy $(P=0.005)$ in this somewhat younger cohort who were considered fit for standard induction therapy.

Overall response rates in azacitidine-treated patients ranged from 17 to $33 \%$ in these analyses, and were higher than response rates with CCR. Interestingly, the difference between azacitidine and CCR overall response rates in AML-MRC (7.5\% improvement with azacitidine) was larger than was the difference seen for all patients in the AZA-AML study (2.7\% in favour of azacitidine) [10]. Similarly, the significant $13.3 \%$ improvement in overall response rate with azacitidine compared with LDAC in LDAC-preselected patients with AML-MRC was much larger than was the difference in response rates for all AML patients in the LDAC preselection group in AZAAML (1.4\% improvement with azacitidine vs LDAC) [21]. Overall response rate with azacitidine in the current AML-MRC analyses was similar to that for all azacitidinetreated patients in AZA-AML (27.8\%) [10]; in contrast, the overall response for LDAC-treated patients with AML-MRC (13.9\%) is lower than that reported for all LDAC-treated patients in AZA-AML (25.9\%) [21]. This suggests that LDAC may be less effective in AML-MRC than in other types of AML.

The safety profile of azacitidine in patients with AMLMRC was similar to that of all azacitidine-treated patients in AZA-AML [10].

\section{Conclusions}

Safety and efficacy results of the current analyses indicate that patients with AML-MRC may fare better if

Table 6 Occurrence of haematological Grade 3-4 adverse events across treatment cycles

\begin{tabular}{|c|c|c|c|c|c|c|c|c|}
\hline \multirow[t]{2}{*}{ Preferred term } & \multicolumn{2}{|l|}{ Cycles 1-2 } & \multicolumn{2}{|l|}{ Cycles 3-4 } & \multicolumn{2}{|l|}{ Cycles 5-6 } & \multicolumn{2}{|l|}{ Cycles 7+ } \\
\hline & $\overline{A Z A}(N=128)$ & $\operatorname{CCR}(N=130)$ & $\mathrm{AZA}(n=89)$ & $\operatorname{CCR}(n=61)$ & $\mathrm{AZA}(n=75)$ & $\operatorname{CCR}(n=25)$ & $\overline{\mathrm{AZA}}(n=57)$ & $\operatorname{CCR}(n=17)$ \\
\hline Any Grade 3-4 TEAE, $n(\%)$ & $83(65)$ & $99(76)$ & $47(53)$ & $35(57)$ & $33(44)$ & $8(32)$ & $37(65)$ & $13(77)$ \\
\hline Febrile Neutropenia, $n(\%)$ & $23(18)$ & $34(26)$ & $7(8)$ & $7(12)$ & $4(5)$ & $3(12)$ & $3(5)$ & $2(12)$ \\
\hline Thrombocytopenia, $n$ (\%) & $24(19)$ & $26(20)$ & $11(12)$ & $8(13)$ & $8(11)$ & 0 & $9(16)$ & 0 \\
\hline Neutropenia, $n(\%)$ & $16(13)$ & $20(15)$ & $10(11)$ & $9(15)$ & $3(4)$ & $2(8)$ & $12(21)$ & $5(29)$ \\
\hline Anaemia, $n(\%)$ & $17(13)$ & $21(16)$ & $3(3)$ & $3(5)$ & $1(1)$ & 0 & $5(9)$ & 0 \\
\hline
\end{tabular}

Safety evaluable patients received at least 1 dose of study drug and had at least 1 post-baseline safety assessment. Patients who received BSC only were included in safety assessments if they had at least 1 post-randomisation safety assessment 
treated with azacitidine rather than with CCR, and more specifically, rather than with LDAC. These data also suggest that diagnostic improvement is needed to more accurately identify patients with preleukaemic haematological disorders, and to recognise multilineage dysplasia. Once AML-MRC is diagnosed, azacitidine may be the treatment of choice for patients who are not candidates for induction chemotherapy or stem cell transplantation.

\section{Abbreviations}

95\%Cl: 95\% confidence interval; AML: Acute myeloid leukaemia; AMLMRC: AML with myelodysplasia-related changes; AML-NOS: AML not otherwise specified; AZA-AML: AZA-AML-001 phase 3 trial; BM: Bone marrow; BSC: Best supportive care; CCR: Conventional care regimens; CR: Complete remission; CRi: CR with incomplete haematologic recovery; ECOG PS: Eastern Cooperative Oncology Group performance status; HR: Hazard ratio; IC: Intensive chemotherapy; IWG: International Working Group; LDAC: Lowdose cytarabine; MDS: Myelodysplastic syndromes; MedDRA: Medical Dictionary for Regulatory Activities; NCCN: National Comprehensive Cancer Network; OS: Overall survival; sAML: Secondary AML; tAML: Therapy-related AML; TEAE: Treatment-emergent adverse event; WBC: White blood cell; WHO: World Health Organization

\section{Acknowledgments}

We thank Nora Tu, PhD, of Celgene Corporation for statistical support for these analyses. Editorial support was provided by Sheila Truten and Kelly Dittmore (Medical Communication Company, Inc.; Wynnewood, PA), funded by Celgene Corporation.

\section{Funding}

This study was funded by Celgene Corporation. The sponsor contributed to study design, collected and analyzed the data, and funded editorial support. The authors interpreted the data and the sponsor reviewed the manuscript for accuracy.

\section{Availability of data and materials}

All data generated for this analysis are included in this published article. Source datasets are available from the corresponding author upon reasonable request. Additional study data are reported in the manuscript by Dombret et al. [10] and at ClinicalTrials.gov (https:/clinicaltrials.gov/ct2/ show/results/NCT01074047).

\section{Authors' contributions}

J.F.S., H. Döhner, J.W., S.S., C.L.B. and H. Dombret designed the study. J.F.S., H. Döhner, A.B., A.W., D.S., J.H.J., R.K., J.C., A.C.S., A.C., C.R., I.S., T.B.d-C., H.K.A., J.F., R.M.S., M.D.M., S.S., C.L.B. and H. Dombret performed the research. J.W. provided statistical analyses and data interpretation. All authors contributed to, revised, and approved manuscript content. All authors gave approval for submission to the journal, and are fully responsible for all content and editorial decisions.

\section{Ethics approval and consent to participate}

The AZA-AML study design was approved by the institutional review boards or independent ethics committees at each study site, and was conducted in accordance with the ethical principles set forth in the Declaration of Helsinki. All patients provided written informed consent before study participation.

\section{Consent for publication}

Not applicable.

\section{Competing interests}

J.F.S.: consultancy for AbbVie, Celgene, Genentech, Gilead, Janssen, Roche Takeda; research funding from AbbVie and Janssen; honoraria from AbbVie, Celgene, Genentech, Gilead, Janssen, Roche, Takeda; speakers' bureau fees from AbbVie, Celgene, Gilead, Janssen, Roche; and advisory board participation for AbbVie, Celgene, Genentech, Gilead, Janssen, Roche, Takeda. H. Döhner: advisory boards (with honoraria) for Agios, Amgen, Astex Pharmaceuticals, Celator, Celgene, Novartis, Roche, Seattle Genetics, Sunesis, and Tolero. A.W.: honoraria from Celgene. D.S.: honoraria and consultancy,
Celgene and Johnson \& Johnson; research funding, Celgene. R.K. consultancy and advisory board participation for Celgene. J.C.: advisory boards and/or speakers' bureaus for Celgene, Janssen and Novartis. A.C.: consultancy and speakers' bureau participation for Celgene. C.R.: honoraria, research funding and consultancy, Celgene, Sunesis, Amgen, and Novartis; honoraria and consulting fees from Pfizer and Jazz Pharmaceuticals. I.S.: consultancy and honoraria from Celgene. H.K.A.: consultancy, honoraria and research funding from Celgene; consultancy, Otsuka. J.F.: consultancy for Celgene. R.M.S.: advisory board participation and consultancy for AbbVie, Actinium, Agios, Amgen, Arog, Astellas, Celgene, Cornerstone, Fujifilm, Janssen, Jazz, Karyopharm, Merck, Novartis, Ono, Orsenix, Otsuka, Pfizer, Roche, Seattle Genetics and Sumitomo; clinical research funding from Agios, Novartis and Arog; DSMB participation for Argenix and Celgene. J.W., S.S and C.L.B. are Celgene employees and own Celgene stock. H. Dombret: consultancy for Amgen and Celgene; honoraria from Agios, Ambit, Amgen, Ariad, Astellas, Celgene, Janssen, Jazz, Karyopharm, Kite Pharma, Menarini, Novartis, Pfizer, Roche/Genentech, Seattle Genetics and Servier; research funding from Amgen, Ariad, Jazz Pharma, Kite Pharma, and Roche/ Genentech. A.B., J.H.J., T.B., M.D.M. and A.C.S.: nothing to disclose.

\section{Publisher's Note}

Springer Nature remains neutral with regard to jurisdictional claims in published maps and institutional affiliations.

\section{Author details}

${ }^{1}$ Department of Haematology, Peter MacCallum Cancer Centre, Locked Bag 1, A'Beckett St, East Melbourne, VIC 8006, Australia. ${ }^{2}$ University of Melbourne, Parkville, Australia. ${ }^{3}$ Universitätsklinikum UIm, Ulm, Germany. ${ }^{4}$ Wroclaw Medical University, Wroclaw, Poland. ${ }^{5}$ Medical University of Lodz, Lodz, Poland. ${ }^{6}$ Algemeen Ziekenhuis Sint-Jan, Brugge, Belgium. ${ }^{7}$ Samsung Medical Center, Sungkyunkwan University School of Medicine, Seoul, South Korea. ${ }^{8}$ Cancer Care Manitoba, Winnipeg, Canada. ${ }^{9}$ Barts Health National Health Service Trust, London, UK. ${ }^{10}$ Princess Margaret Cancer Centre, Toronto, Canada. ${ }^{11}$ Azienda Sanitaria Universitaria Integrata di Udine, Udine, Italy. ${ }^{12}$ Centre Hospitalier Universitaire de Toulouse, Toulouse, France. ${ }^{13}$ University of Alberta Hospital, Edmonton, Canada. ${ }^{14}$ Hospital Central de Asturias, Oviedo, Spain. ${ }^{15}$ University Hospital of Halle, Halle, Germany. ${ }^{16}$ Hospital Universitario Virgen del Rocio/Instituto de BioMedicinia de Sevilla, Sevilla, Spain. ${ }^{17}$ Dana-Farber Cancer Institute, Boston, MA, USA. ${ }^{18}$ Celgene Corporation, Summit, NJ, USA. ${ }^{19}$ Hôpital Saint Louis, Institut Universitaire d’Hématologie, University Paris Diderot, Paris, France.

Received: 22 August 2017 Accepted: 20 November 2017

Published online: 14 December 2017

\section{References}

1. Dohner H, Weisdorf DJ, Bloomfield CD. Acute Myeloid Leukemia. N Engl J Med. 2015;373(12):1136-52. doi:10.1056/NEJMra1406184.

2. Arber DA, Orazi A, Hasserjian R, Thiele J, Borowitz MJ, Le Beau MM, et al. The 2016 revision to the World Health Organization classification of myeloid neoplasms and acute leukemia. Blood. 2016;127(20):2391-405. doi:10.1182/ blood-2016-03-643544

3. Miesner M, Haferlach C, Bacher U, Weiss T, Macijewski K, Kohlmann A et al. Multilineage dysplasia (MLD) in acute myeloid leukemia (AML) correlates with MDS-related cytogenetic abnormalities and a prior history of MDS or MDS/MPN but has no independent prognostic relevance: a comparison of 408 cases classified as "AML not otherwise specified" (AML-NOS) or "AML with myelodysplasia-related changes" (AML-MRC). Blood. 2010;116(15):274251. doi:blood-2010-04-279794 [pii];10.1182/blood-2010-04-279794 [doi].

4. Vardiman JW, Thiele J, Arber DA, Brunning RD, Borowitz MJ, Porwit A et al. The 2008 revision of the World Health Organization (WHO) classification of myeloid neoplasms and acute leukemia: rationale and important changes. Blood. 2009;114(5):937-951. doi:blood-2009-03-209262 [pii];10.1182/blood2009-03-209262 [doi].

5. Weinberg OK, Seetharam M, Ren L, Seo K, Ma L, Merker JD, et al. Clinica characterization of acute myeloid leukemia with myelodysplasia-related changes as defined by the 2008 WHO classification system. Blood. 2009; 113(9):1906-8. doi:10.1182/blood-2008-10-182782.

6. Walter RB, Estey EH. Management of older or unfit patients with acute myeloid leukemia. Leukemia. 2015;29(4):770-5. doi:10.1038/leu.2014.216. 
7. National Comprehensive Cancer Network. NCCN Clinical Practice Guidelines in Oncology. Myelodysplastic Syndromes Version 2.2017. https://www.nccn. org/professionals/physician_gls/pdf/mds.pdf

8. Vidaza prescribing information. Celgene Corporation, Summit, NJ; Revision 8/2016. 2016

9. European Medicines Agency. Vidaza (azacitidine). 2016:EMA/450923/2016.

10. Dombret H, Seymour JF, Butrym A, Wierzbowska A, Selleslag D, Jang JH, et al. International phase 3 study of azacitidine vs conventional care regimens in older patients with newly diagnosed AML with $>30 \%$ blasts. Blood. 2015; 126(3):291-9. doi:10.1182/blood-2015-01-621664.

11. Cheson BD, Bennett JM, Kopecky KJ, Buchner T, Willman CL, Estey EH, et al. Revised recommendations of the international working group for diagnosis, standardization of response criteria, treatment outcomes, and reporting standards for therapeutic trials in acute myeloid leukemia. J Clin Oncol. 2003:21 (24):4642-9. doi:10.1200/JCO.2003.04.036

12. Font P, Loscertales J, Soto C, Ricard P, Novas CM, Martin-Clavero E, et al. Interobserver variance in myelodysplastic syndromes with less than 5\% bone marrow blasts: unilineage vs. multilineage dysplasia and reproducibility of the threshold of 2\% blasts. Ann Hematol. 2015;94(4):56573. doi:10.1007/s00277-014-2252-4.

13. Lindsley RC, Mar BG, Mazzola E, Grauman PV, Shareef S, Allen SL, et al. Acute myeloid leukemia ontogeny is defined by distinct somatic mutations. Blood. 2015;125(9):1367-76. doi:10.1182/blood-2014-11-610543.

14. Dohner H, Estey E, Grimwade D, Amadori S, Appelbaum FR, Buchner T et al. Diagnosis and management of AML in adults: 2017 ELN recommendations from an international expert panel. Blood. 2016:Epub ahead of print. doi:10. 1182/blood-2016-08-733196.

15. Haferlach T, Schoch C, Loffler H, Gassmann W, Kern W, Schnittger S, et al. Morphologic dysplasia in de novo acute myeloid leukemia (AML) is related to unfavorable cytogenetics but has no independent prognostic relevance under the conditions of intensive induction therapy: results of a multiparameter analysis from the German AML cooperative group studies. J Clin Oncol. 2003;21(2):256-65. doi:10.1200/JCO.2003.08.005.

16. Weinberg OK, Pozdnyakova O, Campigotto F, DeAngelo DJ, Stone RM, Neuberg D, et al. Reproducibility and prognostic significance of morphologic dysplasia in de novo acute myeloid leukemia. Mod Pathol. 2015;28(7):965-76. doi:10.1038/modpathol.2015.55.

17. Diaz-Beya M, Rozman M, Pratcorona M, Torrebadell M, Camos M, Aguilar JL, et al. The prognostic value of multilineage dysplasia in de novo acute myeloid leukemia patients with intermediate-risk cytogenetics is dependent on NPM1 mutational status. Blood. 2010;116(26):6147-8. doi:10.1182/blood2010-09-307314.

18. Devillier R, Mansat-De Mas V, Gelsi-Boyer V, Demur C, Murati A, Corre J, et al. Role of ASXL1 and TP53 mutations in the molecular classification and prognosis of acute myeloid leukemias with myelodysplasia-related changes. Oncotarget. 2015;6(10):8388-96. doi:10.18632/oncotarget.3460.

19. Burnett AK, Milligan D, Prentice AG, Goldstone AH, McMullin MF, Hills RK, et al. A comparison of low-dose cytarabine and hydroxyurea with or without alltrans retinoic acid for acute myeloid leukemia and high-risk myelodysplastic syndrome in patients not considered fit for intensive treatment. Cancer. 2007; 109(6):1114-24. doi:10.1002/cncr.22496 [doi].

20. Lancet JE, Uy GL, Cortes JE, Newell LF, Lin TL, Ritchie EK et al. Final results of a phase III randomized trial of CPX-351 versus $7+3$ in older patients with newly diagnosed high risk (secondary) AML. J Clin Oncol. 2016;34:Abstract 7000

21. Seymour JF, Dohner H, Kumar R, Stone RM, Wierzbowska A, Bernal Del Castillo T et al. Overall survival (OS) and clinical outcomes in older patients with acute myeloid leukemia (AML) treated with azacitidine (AZA) or lowdose cytarabine (LDAC) in the AZA-AML-001 study. Presented at the 20thCongress of the European Hematology Association (EHA). 2015; June 11-14, 2015, Vienna, Austria:Poster E9545.

\section{Submit your next manuscript to BioMed Central and we will help you at every step:}

- We accept pre-submission inquiries

- Our selector tool helps you to find the most relevant journal

- We provide round the clock customer support

- Convenient online submission

- Thorough peer review

- Inclusion in PubMed and all major indexing services

- Maximum visibility for your research

Submit your manuscript at www.biomedcentral.com/submit
Biomed Central 